\title{
LAGRANGIAN FIBRATIONS ON HYPERKÄHLER FOURFOLDS
}

\author{
DANIEL GREB, CHRISTIAN LEHN, AND SÖNKE ROLLENSKE
}

\begin{abstract}
Answering the strong form of a question posed by Beauville, we give a short geometric proof that any hyperkähler fourfold containing a Lagrangian subtorus $L$ admits a holomorphic Lagrangian fibration with fibre $L$.
\end{abstract}

\section{INTRODUCTION}

Let $X$ be a hyperkähler manifold, that is, a compact, simply-connected Kähler manifold $X$ such that $H^{0}\left(X, \Omega_{X}^{2}\right)$ is spanned by a holomorphic symplectic form $\sigma$. By work of Matsushita it is well-known that the only possible non-trivial holomorphic maps from $X$ to a lower-dimensional complex space are Lagrangian fibrations, see section 2. Moreover, a special version of the so-called Hyperkähler SYZ-conjecture asserts that any hyperkähler manifold can be deformed to a hyperkähler manifold admitting a Lagrangian fibration.

Hence, it is an important problem to find geometric conditions on a given hyperkähler manifold that guarantee the existence of a Lagrangian fibration; here we address a question posed by Beauville [Bea11, Sect. 1.6]:

Question B - Let X be a hyperkähler manifold and L a Lagrangian torus in X. Is L a fibre of a (meromorphic) Lagrangian fibration $f: X \rightarrow B$ ?

In our previous article [GLR11] it is shown that Question B has a positive answer in case $X$ is non-projective. Moreover, for any hyperkähler manifold that admits an almost holomorphic Lagrangian fibration, a further hyperkähler manfold, birational to the first one, is found, on which the Lagrangian fibration becomes holomorphic.

The approach to the projective case of Beauville's question pursued here is based on a detailed study of the deformation theory of $L$ in $X$. For this, consider the component $\mathfrak{B}$ of the Barlet space that contains $[L]$ together with its universal family and the evaluation map to $X$ :

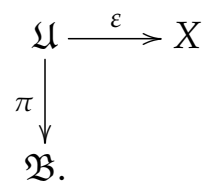

It was shown in [GLR11, Lemma 3.1] that $\varepsilon$ is surjective and generically finite, and that $X$ admits an almost holomorphic Lagrangian fibration if and only if $\operatorname{deg}(\varepsilon)=1$.

If the degree of $\varepsilon$ is strictly bigger than one, some deformations of $L$ intersect $L$ in unexpected ways. In order to deal with this, we introduce the notion of L-reduction: for each projective hyperkähler manifold containing a Lagrangian torus there exists a projective variety $\mathfrak{T}$ and a rational map $\varphi_{L}: X \rightarrow \mathfrak{T}$, uniquely defined up to birational equivalence, whose fibre through a general point $x$ coincides with the connected component of the intersection of all deformations of $L$ through $x$. In this situation, we say that $X$ is $L$-separable if $\varphi_{L}$ is birational, and prove the following result:

2010 Mathematics Subject Classification. 53C26, 14D06, 14E30, 32G10, 32 G05.

Key words and phrases. hyperkähler manifold, Lagrangian fibration. 
Theorem 3.5 - Let X be a projective hyperkähler manifold and $L \subset X$ a Lagrangian subtorus. Then $X$ admits an almost holomorphic fibration with strong fibre $L$ if and only if $X$ is not $L$ separable.

If $X$ is a hyperkähler fourfold, then we can exclude the case that $X$ is $L$-separable by symplectic linear algebra. Moreover, based upon the rather explicit knowledge about the birational geometry of hyperkähler fourfolds we obtain a positive answer to the strongest form of Beauville's question:

Theorem 5.1 - Let X be a four-dimensional hyperkähler manifold containing a Lagrangian torus $L$. Then $X$ admits a holomorphic Lagrangian fibration with fibre $L$.

At the Moscow conference "Geometric structures on complex manifolds" Ekaterina Amerik brought to our attention that she had independently shown a related result, based on an observation from [AC08], to the effect that in dimension four every projective hyperkähler manifold containing a Lagrangian subtorus $L$ admits an almost holmorphic Lagrangian fibration with fibre $L[\mathrm{~A} 11] .^{1}$

Acknowledgements. The authors want to thank Daniel Huybrechts for his interest in our work and for several stimulating discussions. We are grateful to Ekaterina Amerik for communicating to us the observation contained in Lemma 5.3, which greatly simplified our previous argument. The second author thanks Laurent Manivel for stimulating discussions, in particular, for pointing out Remark 5.4. The third author thanks Misha Verbitsky for an invitation to Moscow.

The support of the DFG through the SFB/TR 45, Forschergruppe 790, and the third author's Emmy-Noether project was invaluable for the success of the collaboration. The first author gratefully acknowledges the support of the Baden-Württemberg-Stiftung via the "Eliteprogramm für Postdoktorandinnen und Postdoktoranden". The second author acknowledges the support by the CNRS and the Institut Fourier.

\section{PRELIMINARIES AND SETUP OF NOTATION}

\subsection{Lagrangian fibrations.}

Definition 2.1 - Let $X$ be a hyperkähler manifold. A Lagrangian fibration on $X$ is a holomorphic map $f: X \rightarrow B$ with connected fibres onto a normal complex space $B$ such that every irreducible component of the reduction of every fibre of $f$ is a Lagrangian subvariety of $X$.

Due to fundamental results of Matsushita it is known that any fibration on a hyperkähler manifold is automatically Lagrangian:

Theorem 2.2 ([Mat99, Mat00, Mat01, Mat03]) — Let X be a hyperkähler manifold of dimension $2 n$. If $f: X \rightarrow B$ is a morphism with connected fibres to a normal complex space $B$ with $0<$ $\operatorname{dim} B<\operatorname{dim} X$, then $f$ is a Lagrangian fibration. In particular, $f$ is equidimensional and $\operatorname{dim} B=n$. Furthermore, every smooth fibre of $f$ is a complex torus.

\footnotetext{
${ }^{1}$ After this article was written, Jun-Muk Hwang and Richard Weiss have posted a proof of the projective case of the weak form of Beauville's question, producing an almost holomorphic Lagrangian fibration on any projective $2 n$-dimensional hyperkähler manifold containing a Lagrangian torus, see [HW12]. Their argument has two parts: one is geometric and one is concerned with abstract group theory. In contrast, our answer to the strong form of Beauville's question, Theorem 5.1, is purely geometric, uses global arguments in addtion to local deformation theoretic ones, and uses symplectic linear algebra in place of their grouptheoretic arguments.
} 
2.2. Meromorphic maps. Let $X$ be a normal complex space, $Y$ a compact complex space, and $f: X \rightarrow Y$ a meromorphic map. Let

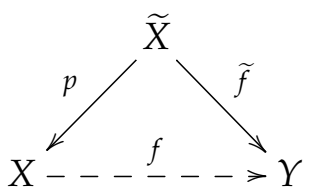

be a resolution of the indeterminacies of $f$. The fibre $F_{y}$ of $f$ over a point $y \in Y$ is defined to be $F_{y}:=p\left(\tilde{f}^{-1}(y)\right)$. This is independent of the chosen resolution.

Recall that a meromorphic map $f: X \rightarrow Y$ as above is called almost holomorphic if there is a Zariski-open subset $U \subset Y$ such that the restriction $\left.f\right|_{f^{-1}(U)}: f^{-1}(U) \rightarrow U$ is holomorphic and proper. A strong fibre of an almost holomorphic map $f$ is a fibre of $\left.f\right|_{f^{-1}(U)}$.

Let $X$ be a normal algebraic variety, $B$ a complete algebraic variety, and $f: X \rightarrow B$ an almost holomorphic rational map. If $A$ is a divisor on $B$, then its pullback via $f$ is defined either geometrically as the closure of the pullback on the locus where $f$ is holomorphic, or on the level of locally free sheaves as $f^{*} \mathcal{O}_{B}(A):=\left(p_{*} \widetilde{f}^{*} \mathcal{O}_{B}(A)\right)^{\vee \vee}$, where $p: \widetilde{X} \rightarrow X$ is a resolution of indeterminacies as in diagram (1).

2.3. Deformations of Lagrangian subtori. The starting point for our approach to Beauville's question is the deformation theory of a Lagrangian subtorus $L$ in a hyperkähler manifold $X$. We quickly recall the relevant results from [GLR11, Sects. 2 and 3].

The Barlet space $\mathfrak{B}(X)$ of $X$ (or Chow scheme in the projective setting) parametrises compact cycles in $X$ and it turns out (see (i) of Lemma 2.3 below) that there is a unique irreducible component $\mathfrak{B}$ of $\mathfrak{B}(X)$ containing the point $[L]$. Denoting by $\mathfrak{U}$ the graph of the universal family over $\mathfrak{B}$ and by $\Delta$ the discriminant locus of $\mathfrak{B}$, i.e., the set of points parametrising singular elements in the family $\mathfrak{B}$, we obtain the following diagram.

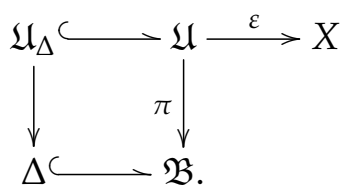

A detailed analysis of the maps in diagram (2) shows that a small étale or analytic neighbourhood of $L$ in $X$ fibres over a neighbourhood of $[L]$ in $\mathfrak{B}$. More precisely, we have the following result.

Lemma 2.3 ([GLR11, Lem. 3.1]) - Let $X$ be a hyperkähler manifold of dimension $2 n$ and let $L$ be a Lagrangian subtorus of $X$. Then, the following holds.

(i) The Barlet space $\mathfrak{B}(X)$ is smooth of dimension $n$ near $[L]$. In particular, $[L]$ is contained in a unique irreducible component $\mathfrak{B}$ of $\mathfrak{B}(X)$ and $\mathfrak{U}$ is smooth of dimension $2 n$ near $\pi^{-1}([L])$.

(ii) The morphism $\varepsilon$ is finite étale along smooth fibres of $\pi$. In particular, a sufficiently small deformation of $L$ is disjoint from $L$ and there are no positive-dimensional families of smooth fibres through a general point $x \in X$.

(iii) If $\left[L^{\prime}\right] \in \mathfrak{B}$ with smooth $L^{\prime}$, then $L^{\prime}$ is a Lagrangian subtorus of $X$.

Remark 2.4 - We remark two simple but useful consequences of Lemma 2.3.

(i) The locus $X_{\Delta}:=\varepsilon\left(\mathfrak{U}_{\Delta}\right)$ is the locus of points $x \in X$ such that there is a singular deformation of $L$ passing through $x$. By dimension reasons it is a proper subset of $X$ and by Lemma 2.3 (ii) the map $\varepsilon$ is finite and étale on the preimage of $X \backslash X_{\Delta}$. 
(ii) Statement (ii) implies in particular that for any two points $[L],[M] \in \mathfrak{B}$ the intersection product $[L] .[M]$ as cycles in $X$ vanishes. It is therefore impossible for members of the family $\mathfrak{B}$ to intersect in a finite number of points.

2.4. Almost holomorphic Lagrangian fibrations and Barlet spaces. The following result relates the deformation theory of $L$ in $X$ discussed above to our question about globally defined almost holomorphic Lagrangian fibrations.

Lemma 2.5 ([GLR11, Lem. 3.2]) - Let X be a hyperkähler manifold containing a Lagrangian subtorus L. Then $X$ admits an almost holomorphic Lagrangian fibration with strong fibre $L$ if and only if the evaluation map $\varepsilon$ in diagram (2) is bimeromorphic.

If $\varepsilon$ is birational, then $\pi \circ \varepsilon^{-1}$ is the desired almost holomorphic fibration (up to normalisation of $\mathfrak{B}$ ). For the other direction one uses the Barlet space of a resolution of indeterminacies.

\section{L-REDUCTION AND L-SEPARABLE MANIFOLDS}

Let $X$ be a projective hyperkähler manifold containing a Lagrangian subtorus $L$. In this section we start our analysis of the maps in the associated diagram (2). Recall from Lemma 2.5 above that in order to answer Beauville's question positively we have to show that the evaluation map $\varepsilon$ is birational.

3.1. L-reduction. Here, we construct a meromorphic map associated with the covering family $\left\{L_{t}\right\}_{t \in \mathfrak{B}}$. Generically, this map is a quotient map for the meromorphic equivalence relation defined by the family $\left\{L_{t}\right\}$, i.e., generically it identifies those points in $X$ that cannot be separated by members of $\left\{L_{t}\right\}$.

3.1.1. Construction of the L-reduction. We work in the setup summarised in diagram (2). We set $\mathfrak{U}_{\text {reg }}:=\varepsilon^{-1}\left(X \backslash X_{\Delta}\right)$. Recall from Remark 2.4 that the map

$$
\left.\varepsilon\right|_{\mathfrak{U}_{\text {reg }}}: \mathfrak{U}_{\mathrm{reg}} \rightarrow X \backslash X_{\Delta}
$$

is finite étale; we denote its degree by $d$.

The map $\left.\varepsilon\right|_{\mathfrak{L}_{\text {reg }}}$ induces a morphism $X \backslash X_{\Delta} \rightarrow \operatorname{Sym}^{d}\left(\mathfrak{U}_{\text {reg }}\right)$. Composing this map with the natural morphism $\operatorname{Sym}^{d}\left(\mathfrak{U}_{\text {reg }}\right) \rightarrow \operatorname{Sym}^{d}(\mathfrak{B})$ induced by $\pi: \mathfrak{U} \rightarrow \mathfrak{B}$, we construct a morphism $X \backslash X_{\Delta} \rightarrow \operatorname{Sym}^{d}(\mathfrak{B})$. This morphism naturally extends to a rational map $\psi: X \rightarrow \operatorname{Sym}^{d}(\mathfrak{B})$. Let

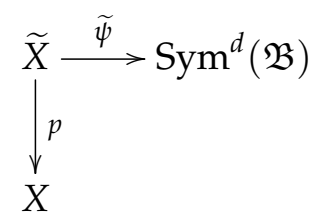

be a resolution of singularities of the indeterminacies of $\psi$ with $\widetilde{X}$ nonsingular. The Stein factorisation of $\widetilde{\psi}$ then yields the following diagram.

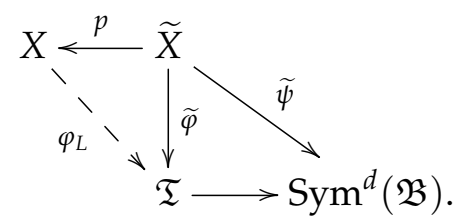

Here, $\varphi_{L}=\tilde{\varphi} \circ p^{-1}: X \rightarrow \mathfrak{T}$ is the rational map induced by $\widetilde{\varphi}$. Noting that $\varphi_{L}: X \rightarrow \mathfrak{T}$ is unique up to birational equivalence, and hence canonically associated with the pair $(X, L)$, we call it the L-reduction of $X$. 
Remark 3.1 - For every point $x \in X \backslash X_{\Delta}$ there are exactly $d$ pairwise distinct smooth tori $L_{1}, \ldots, L_{d}$ in the family $\left\{L_{t}\right\}_{t \in \mathfrak{B}}$ containg $x$. By construction, $\varphi_{L}$ is defined at $x$ and maps it to the class of $\left(\left[L_{1}\right], \ldots,\left[L_{d}\right]\right)$ in $\operatorname{Sym}^{d}(\mathfrak{B})$.

3.1.2. First properties of the L-reduction. The following set-theoretical assertion is an immediate consequence of the construction of $\varphi_{L}$.

Lemma 3.2 - The fibre of $\varphi_{L}$ through a point $x \in X \backslash X_{\Delta}$ coincides with the connected component of

containing $x$.

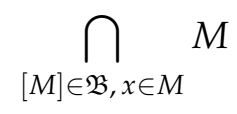

Proof. If $x \in X \backslash X_{\Delta}$, then $\varepsilon$ is étale in every point of the preimage $\varepsilon^{-1}(x)$. Thus the image $\pi\left(\varepsilon^{-1}(x)\right)=\left\{\left[L_{1}\right], \ldots,\left[L_{d}\right]\right\}$ consists of the points in $\mathfrak{B}$ that parametrise the $d$ pairwise distinct subtori in $X$ through $x$. In particular, the meromorphic map $\psi: X \rightarrow \operatorname{Sym}^{d}(\mathfrak{B})$ is defined at $x$ and its fibre is

$$
\psi^{-1}(\psi(x))=\bigcap_{i} L_{i} .
$$

After taking the Stein factorisation, the fibre of $\varphi_{L}$ is the component of (3) through $x$, as claimed.

Lemma 3.3 - Let X be a projective hyperkähler manifold containing a Lagrangian subtorus L. Then the L-reduction $\varphi_{L}: X \rightarrow T$ is almost holomorphic.

Proof. Let $\operatorname{dom}\left(\varphi_{L}\right)$ be the domain of definition of $\varphi_{L}$, and let $Z:=X \backslash \operatorname{dom}\left(\varphi_{L}\right)$ be the locus where $\varphi_{L}$ is not defined. We have to show that the general fibre of $\varphi_{L}$ does not intersect $Z$.

Aiming for a contradiction, suppose that for a general $x_{0} \in X \backslash X_{\Delta}$ the fibre $F_{x_{0}}$ of $\varphi_{L}$ through $x_{0}$ intersects $Z$ nontrivially. Recall from item (i) of Remark 2.4 that $X_{\Delta}=\varepsilon\left(\mathfrak{U}_{\Delta}\right)$ is the locus swept out by singular deformations of $L$ and from Remark 3.1 that $\varphi_{L}$ is holomorphic on $X \backslash X_{\Delta}$. Take a point $z \in F_{x_{0}} \cap Z$. Consider the graph $X^{\prime} \subset X \times \mathfrak{T}$ of $\varphi_{L}$ with projections $p: X^{\prime} \rightarrow X$ and $\varphi_{L}^{\prime}: X^{\prime} \rightarrow \mathfrak{T}$. As explained for example in [Deb01, Sect. 1.39], the closed subset $Z$ can be described as

$$
Z=\left\{x \in X \mid \operatorname{dim} p^{-1}(x)>0\right\} .
$$

As $X$ is normal and $p$ is birational, $p$ has connected fibres. Thus, the variety $C^{\prime}:=$ $\varphi_{L}^{\prime}\left(p^{-1}(z)\right)$ is connected. We list some further properties of $C^{\prime}$ :

(i) $\operatorname{dim} C^{\prime}>0$, because $\operatorname{dim} p^{-1}(z)>0$ and $X^{\prime}$ is the graph of $\varphi_{L}$,

(ii) $\varphi_{L}\left(x_{0}\right) \in C^{\prime}$ as $z \in F_{x_{0}}$,

(iii) the point $z$ is contained in all fibres over points in $C^{\prime}$.

Suppose for the moment that we had a diagram

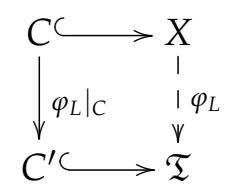

such that $C$ is connected, $x_{0} \in C$ and $\left.\varphi_{L}\right|_{C}$ is a local isomorphism at the point $x_{0}$. We claim that this would produce a contradiction. Namely, let $L_{1}, \ldots, L_{d}$ be the $d=\operatorname{deg} \varepsilon$ pairwise distinct tori in the family $\left\{L_{t}\right\}_{t \in \mathfrak{B}}$ containing $x_{0}$. Since $C$ is connected and $C \not \subset F_{x_{0}}$ by item (i) above, Lemma 3.2 implies that there exists $k \in\{1, \ldots, d\}$ such that $C \not \subset L_{k}$. By 
Lemma 2.3, small deformations of $L_{k}$ constitute a fibration in an analytic neighbourhood of $x_{0}$. Thus, for all points $y \in C \backslash\left\{x_{0}\right\}$ sufficiently close to $x_{0}$ there is a small deformation $L_{y}$ of $L_{k}$ with $y \in L_{y}$ and

$$
L_{y} \cap L_{k}=\varnothing .
$$

On the other hand, item (iii) above and Lemma 3.2 imply that

$$
z \in F_{y} \cap F_{x_{0}} \subset L_{y} \cap L_{k}
$$

which in view of (5) is absurd.

It remains to find the variety $C$. We observe that it suffices to construct $C$ in an Euclidean open neighbourhood of $x_{0}$. Invoking the generality assumption on $x_{0}$ and the implicit function theorem we find a small neighbourhood $U \ni x_{0}$ such that the restriction $\varphi_{L}: U \rightarrow V:=\varphi_{L}(U)$ is a trivial holomorphic fiber bundle. In particular, $V \subset \mathfrak{T}$ is open and there is a section $C \subset U$ for the subvariety $C^{\prime} \cap V$. The only remaining property to be fulfilled is connectedness of $C^{\prime} \cap V$ and $C$. This may be achieved by shrinking $V$ and $U$, and so we conclude the proof.

Definition 3.4 - A projective hyperkähler manifold $X$ containing a Lagrangian subtorus $L$ is called $L$-separable if its $L$-reduction $\varphi_{L}: X \rightarrow \mathfrak{T}$ is birational.

\subsection{Lagrangian fibrations on non- $L$-separable manifolds.}

Theorem 3.5 - Let X be a projective hyperkähler manifold and $L \subset X$ a Lagrangian subtorus. Then $X$ admits an almost holomorphic fibration with strong fibre $L$ if and only if $X$ is not $L$ separable.

As a consequence of this result we can reformulate Beauville's question in the following way.

Question B" - Does there exist a projective hyperkähler manifold X together with a Lagrangian subtorus $L$ such that $X$ is L-separable?

Proof of Theorem 3.5. If $X$ is not $L$-separable, the $L$-reduction $\varphi_{L}: X \rightarrow \mathfrak{T}$ is an almost holomorphic map (Lemma 3.3) such that $0<\operatorname{dim} \mathfrak{T}<\operatorname{dim} X$. Thus by [GLR11, Thm. 6.7], the map $\varphi_{L}$ is an almost holomorphic Lagrangian fibration on $X$. By the description of the general fibre of the $L$-reduction (Lemma 3.2), the torus $L$ is a strong fibre of $\varphi_{L}$.

If conversely $f: X \rightarrow B$ is an almost holomorphic Lagrangian fibration with strong fibre $L$, then through the general point there is a unique Lagrangian subtorus in $\mathfrak{B}$ and the $L$-reduction coincides with the rational map $\pi \circ \varepsilon^{-1}: X \rightarrow \mathfrak{B}$. In particular, $X$ is not $L$-separable.

\section{INTERSECTIONS OF LAGRANGIAN SUBTORI}

As before, let $X$ be a projective hyperkähler manifold containing a Lagrangian subtorus $L$. In this section we study a neighbourhood of $L$ in $X$ more closely, which leads to several results about the geometry of intersections of different members in the family $\mathfrak{B}$ of deformations of $L$. We are going to use the notation and the results of Section 2.3 throughout.

By Lemma $2.3, \mathfrak{B}$ is smooth at $[L]$ and we can find a neighbourhood $V$ of $[L]$ such that the restriction $\varepsilon_{\mathfrak{U}_{V}}: \mathfrak{U}_{V} \rightarrow X$ of the evaluation map to the preimage $\mathfrak{U}_{V}:=\pi^{-1}(V)$ embeds $\mathfrak{U}_{V}$ into $X$. We may thus consider $\mathfrak{U}_{V}$ as an open subset of $X$. The intersection of $\mathfrak{U}_{V}$ with a submanifold $M \subset X$ is depicted in Figure 1. 


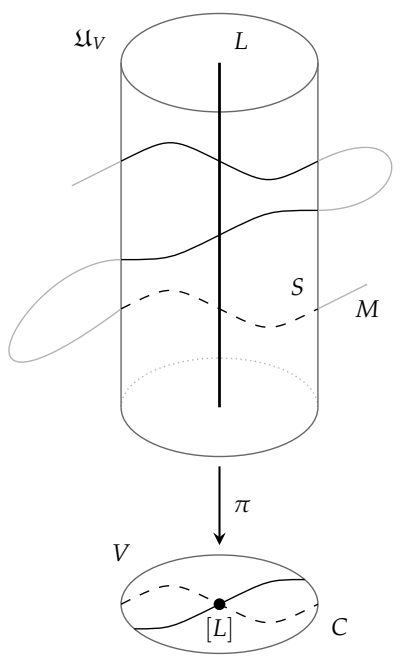

FIGURE 1. The neighbourhood $\mathfrak{U}_{V}$ of $L$ and its projection to $V \subset \mathfrak{B}$.

Lemma 4.1 - Let $M \subseteq X$ be a smooth and proper submanifold, and $L \subset X$ a smooth Lagrangian torus that intersects $M$ nontrivially. Then a generic small deformation of $L$ has smooth intersection with $M$.

Proof. We continue to use the notation introduced above. Since $\mathfrak{U}_{V}$ is open in $X$, the intersection $M \cap \mathfrak{U}_{V}$ is smooth. Furthermore, the map $\left.\pi\right|_{M \cap \mathfrak{U}_{V}}: M \cap \mathfrak{U}_{V} \rightarrow V$ is proper, because $\pi$ is proper and $M$ is compact. We can therefore apply the theorem on generic smoothness to $\left.\pi\right|_{M \cap \mathfrak{U}_{V}}$ which proves the result.

Proposition 4.2 - Let $M \subseteq X$ be a compact submanifold and $L \subseteq X$ be a general Lagrangian subtorus, such that $L \cap M \neq \varnothing$. Then $N_{L \cap M / M}$ is trivial. If $M$ is a complex torus, then $L \cap M$ is a disjoint union of tori.

Proof. As $L$ is general, the intersection $L \cap M$ is smooth by Lemma 4.1. Moreover, both statements can be verified by looking at one connected component of $L \cap M$ at a time. We invoke the notation introduced in the beginning of this section, and let $T$ be a connected component of $L \cap M$. If $V$ is sufficently small, then the inclusion $L \cap M \hookrightarrow \mathfrak{U}_{V} \cap M$ induces a one-to-one correspondence of their respective connected components. Let $S$ be the unique component of $\mathfrak{U}_{V} \cap M$ corresponding to $T$. By generality of $L$ we may assume that $\left.\pi\right|_{S}$ is a smooth map, thus $C:=\pi(S) \subset V$ is smooth of dimension $n-\operatorname{dim} T$ near $[L]$. Moreover, $C$ parametrizes those small deformations of $L$ that induce a flat deformation of $T$ inside $M$. Corresponding to the family $S \rightarrow C$ we thus obtain a classyfying map $\chi: C \rightarrow \mathscr{D}(M)$ from $C$ to the Douady-space of $M$.

On the level of tangent spaces we have $T_{C}([L]) \subset T_{\mathfrak{B}}([L])=H^{0}\left(L, N_{L / X}\right)$, where the last equality comes from the Hilbert-Chow morphism, compare [GLR11, Lem. 3.1]. The morphism $\chi$ induces a map $\chi_{*}: T_{C}([L]) \rightarrow H^{0}\left(T, N_{T / M}\right)$. But small deformations of $T$ inside $M$ induced by deformations of $L$ are disjoint from $T$ by Lemma 2.3 (ii). Thus the map $\chi_{*}$ is injective, and the image of $T_{C}([L])$ consists of nowhere vanishing sections. For dimension reasons these sections generate the normal bundle of $T$ in $M$, and consequently $N_{T / M}$ is trivial, as claimed.

If $M$ is a torus as well, then $\left.T_{M}\right|_{T}$ is likewise trivial. So, by the normal bundle sequence

$$
\left.0 \longrightarrow T_{T} \longrightarrow T_{M}\right|_{T} \longrightarrow N_{T / M} \longrightarrow 0
$$

the tangent bundle $T_{T}$ is trivial, and thus $T$ is a complex torus. 
Based on the preceeding result we can now refine the observation in Remark 2.4(ii):

Lemma 4.3 - Let $X$ be a four-dimensional hyperkähler manifold. Let $L$ and $M$ be two Lagrangian tori intersecting smoothly, and set $I=L \cap M$. Then, $I$ is a finite disjoint union of elliptic curves.

Proof. It remains to exclude the existence of zero-dimensional connected components of I. By general Lagrangian intersection theory, see for example [BF09, Introduction], we have

$$
[L] \cdot[M]=\chi(I) .
$$

However, this already implies the claim, since by Proposition 4.2 above, any positive dimensional component of $I$ is a smooth elliptic curve, contributing zero to the Euler characteristic $\chi(I)$.

Corollary 4.4 - Let X be a four-dimensional projective hyperkähler manifold, L a Lagrangian subtorus. Assume that X is L-separable. Then, the evaluation map $\varepsilon$ in Diagram (2) has degree at least three.

Proof. As $X$ is assumed to be $L$-separable, Theorem 3.5 and Lemma 2.5 imply that $\varepsilon$ is not birational. It remains to exclude the case $\operatorname{deg} \varepsilon=2$. By Lemma 3.2, the L-separability means that at a general point $x \in X$ the connected component of $\bigcap_{[M] \in \mathfrak{B}, x \in M} M$ is just $x$. If $\operatorname{deg} \varepsilon=2$ there are just two tori in $\mathfrak{B}$ containing $x$, say $M_{1}$ and $M_{2}$. As $x$ was general, Lemma 4.1 tells us that $M_{1} \cap M_{2}$ is smooth. Then Lemma 4.3 contradicts the fact that the connected component of $M_{1} \cap M_{2}$ containing $x$ is $\{x\}$.

\section{HYPERKÄHLER FOURFOLDS}

Using the results from the last section we can now prove our main result which gives the strongest possible positve answer to Beauville's question:

Theorem 5.1 - Let X be a four-dimensional hyperkähler manifold containing a Lagrangian torus L. Then $X$ admits a holomorphic Lagrangian fibration with fibre $L$.

Remark 5.2 - We are grateful to E. Amerik for communicating the following linear algebra observation to us which serves to exclude $L$-separable manifolds $X \supset L$ in dimension four. This greatly simplified a previous deformation-theoretic argument.

Lemma 5.3 - Let $V$ be a four-dimensional symplectic vector space with symplectic form $\sigma$, and let $W_{1}, W_{2}, W_{3} \subset V$ be three Lagrangian subspaces satisfying $\operatorname{dim} W_{i} \cap W_{j}=1$ for all $i \neq j$. Then $W_{1} \cap W_{2} \cap W_{3} \neq\{0\}$.

Proof. Suppose on the contrary that $W_{1} \cap W_{2} \cap W_{3}=\{0\}$ and consider the span $\left\langle W_{1}, W_{2}\right\rangle$. It is of dimension 3 as $\operatorname{dim} W_{1} \cap W_{2}=1$. Moreover, we claim that

$$
W_{3} \subset\left\langle W_{1}, W_{2}\right\rangle \text {. }
$$

Indeed, otherwise we would have $\operatorname{dim} W_{3} \cap\left\langle W_{1}, W_{2}\right\rangle=1$, implying that the intersections $W_{3} \cap\left\langle W_{1}, W_{2}\right\rangle=W_{3} \cap W_{1}=W_{3} \cap W_{2}$ are all one-dimensional, in contradiction to our assumption that $W_{1} \cap W_{2} \cap W_{3}=\{0\}$.

Now, again using $W_{1} \cap W_{2} \cap W_{3}=\{0\}$ we write $\left\langle W_{1}, W_{2}\right\rangle=W_{3} \oplus\left(W_{1} \cap W_{2}\right)$. As $V$ is symplectic and $W_{3}$ is Lagrangian, there is $v \in W_{1} \cap W_{2}$ and $w \in W_{3}$ such that $\sigma(v, w) \neq 0$. According to the inclusion (6) we can write $w=w_{1}+w_{2}$ with $w_{i} \in W_{i}$, so that

$$
0 \neq \sigma(v, w)=\sigma\left(v, w_{1}\right)+\sigma\left(v, w_{2}\right)=0+0=0,
$$

as $W_{1}$ and $W_{2}$ are Lagrangian. Contradiction. 
Remark 5.4 - Lemma 5.3 can also be proven using the following beautiful geometric argument which was explained to us by Laurent Manivel: The Grassmanian of Lagrangian subspaces in $V \cong \mathbb{C}^{4}$ is biholomorphic to the (smooth) intersection of the Plücker quadric $\widetilde{Q} \subset \mathbb{P}\left(\bigwedge^{2} V\right)$ and the linear subspace defined by vanishing of the symplectic form $\sigma: \wedge^{2} V \rightarrow \mathbb{C}$, and hence is a smooth quadric $Q \subset \mathbb{P}^{4}$. The condition $\operatorname{dim} W_{i} \cap W_{j}=1$ means that the line in $\mathbb{P}^{4}$ joining the points $\left[W_{i}\right],\left[W_{j}\right] \in Q$ is contained in $Q$. If the triple intersection $W_{1} \cap W_{2} \cap W_{3}=\{0\}$, then $\left[W_{1}\right],\left[W_{2}\right],\left[W_{3}\right]$ span a plane $P$. But $P \cap Q$ has degree 2 and thus cannot contain a union of 3 lines.

Proposition 5.5 - Let $X$ be a four-dimensional projective hyperkähler manifold containing $a$ Lagrangian torus $L$. Then $X$ is not L-separable.

Proof. Suppose on the contrary that $X$ is $L$-separable. Given a general point $x \in X$, it follows from Lemma 3.2 and Corollary 4.4 that there exists a natural number $d \geq 3$, and $d$ smooth Lagrangian subtori that locally cut out $x$. The point $x$ being general, Lemma 4.1 and Lemma 4.3 imply that there exist two such tori, say $L_{1}$ and $L_{2}$, that intersect in an elliptic curve $E$ at $x$. Any other torus in $\mathfrak{B}$ passing through $x$ either contains $E$ or cuts out a zero-dimensional subscheme.

As a consequence of $L$-separability there exists a lagrangian torus $L_{3}$ containing the point $x$ but not containing $E$, such that the intersection scheme $L_{1} \cap L_{2} \cap L_{3}$ is zerodimensional at the point $x$. Again invoking that $x$ was general, we may assume that the intersections $L_{1} \cap L_{3}, L_{2} \cap L_{3}$ and $L_{1} \cap L_{2} \cap L_{3}$ are smooth at $x$. Consequently, the three Lagrangian subspaces $W_{i}:=T_{L_{i}, x} \subset T_{X, x}$ satisfy the assumptions of Lemma 5.3. It follows that $W_{1} \cap W_{2} \cap W_{3} \neq\{0\}$, contradicting our choice of $L_{1}, L_{2}, L_{3}$. Therefore, $X$ cannot be $L$-separable.

Proof of Theorem 5.1. If $X$ is not projective, we are done by [GLR11, Thm. 4.1], so we may assume $X$ to be projective. By Proposition 5.5, $X$ is not $L$-separable and hence admits an almost holomorphic Lagrangian fibration $f: X \rightarrow B$ by Theorem 3.5. It remains to show that the existence of an almost holomorphic Lagrangian fibration implies the existence of a holomorphic one, which will be done in Lemma 5.6 below.

Lemma 5.6 - Let $f: X \rightarrow B$ be an almost holomorphic Lagrangian fibration on a projective hyperkähler fourfold. Then there exists a birational modification $\psi: B \rightarrow B^{\prime}$ such that $\psi \circ$ $f: X \rightarrow B^{\prime}$ is a holomorphic Lagrangian fibration.

The proof of Lemma 5.6 rests on the explicit knowledge of the birational geometry of hyperkähler fourfolds. For this we recall the notion of Mukai flop: Assume that a hyperkähler fourfold $X$ contains a smooth subvariety $P \cong \mathbb{P}^{2}$. If we blow up $P$, the exceptional divisor is isomorphic to the projective bundle $\mathbb{P}\left(\Omega_{\mathbb{P}^{2}}^{1}\right)$, and it is well known that it can be blown down in the other direction to yield another hyperkähler manifold $X^{\prime}$. The resulting birational transformation $X \rightarrow X^{\prime}$ is called the Mukai flop at $P$.

Proof of Lemma 5.6. By [GLR11, 6.2] there exists a holomorphic model for $f$, that is, a Lagrangian fibration $f^{\prime}: X^{\prime} \rightarrow B^{\prime}$ on a possibly different hyperkähler manifold $X^{\prime}$ and a diagram

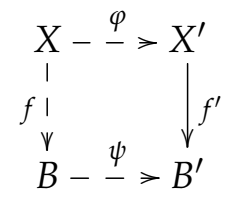


with birational horizontal arrows such that $\varphi$ is an isomorphism near the general fibre of $f$.

We claim that the composition $f^{\prime} \circ \varphi=\psi \circ f$ is holomorphic and thus a Lagrangian fibration on $X$. To see this first note that by [WW03, Thm. 1.2] the map $\varphi$ factors as a finite composition of Mukai flops, so by induction we may assume that $\varphi^{-1}$ is the simultaneous Mukai flop of a disjoint union of embedded projective planes $\mathbb{P}^{2} \cong P_{i} \subset X^{\prime}$.

As $\varphi$ is holomorphic near a general fibre of $f^{\prime}$, none of the $P_{i}^{\prime}$ 's can intersect the general fibre. Thus $f^{\prime}\left(P_{i}\right)$ is a proper subset of $B^{\prime}$ and hence of dimension at most 1 . Since there is no non-constant map from $\mathbb{P}^{2}$ to a curve, $f^{\prime}\left(P_{i}\right)$ is a single point. In other words, the locus of indeterminacy of $\varphi^{-1}$ is contained in the fibres of $f^{\prime}$, and thus the composition $f^{\prime} \circ \varphi$ remains holomorphic.

\section{REFERENCES}

[A11] Ekaterina Amerik. A remark on a question of Beauville about lagrangian fibrations, arXiv:1110.2852, 2011. (cited on p. 2)

[AC08] Ekaterina Amerik and Frédéric Campana. Fibrations méromorphes sur certaines variétés à fibré canonique trivial. Pure Appl. Math. Q., 4(2, part 1):509-545, 2008. (cited on p. 2)

[Bea11] Arnaud Beauville. Holomorphic symplectic geometry: a problem list. In Complex and Differential Geometry, Conference held at Leibniz Universität Hannover, September 14 - 18, 2009, volume 8 of Springer Proceedings in Mathematics. Springer, 2011, preprint arXiv:1002.4321. (cited on p. 1)

[BF09] Kai Behrend and Barbara Fantechi. Gerstenhaber and Batalin-Vilkovisky structures on Lagrangian intersections. In Algebra, arithmetic, and geometry: in honor of Yu. I. Manin. Vol. I, volume 269 of Progr. Math., pages 1-47. Birkhäuser Boston Inc., Boston, MA, 2009. (cited on p. 8)

[Deb01] Olivier Debarre. Higher-Dimensional Algebraic Geometry. Universitext. Springer-Verlag, New York, 2001. (cited on p. 5)

[GLR11] Daniel Greb, Christian Lehn, and Sönke Rollenske. Lagrangian fibrations on hyperkähler manifolds - On a question of Beauville. Preprint arXiv:1105.3410, 2011, to appear in Ann. Sci. Éc. Norm. Supér. (cited on p. 1, 3, 4, 6, 7, 9)

[HW12] Jun-Muk Hwang and Richard Weiss. Webs of Lagrangian Tori in Projective Symplectic Manifolds, Invent. Math., Online First, DOI:10.1007/s00222-012-0407-2 (cited on p. 2)

[Mat99] Daisuke Matsushita. On fibre space structures of a projective irreducible symplectic manifold. Topology, 38(1):79-83, 1999. (cited on p. 2)

[Mat00] Daisuke Matsushita. Equidimensionality of Lagrangian fibrations on holomorphic symplectic manifolds. Math. Res. Lett., 7(4):389-391, 2000. (cited on p. 2)

[Mat01] Daisuke Matsushita. Addendum: “On fibre space structures of a projective irreducible symplectic manifold" [Topology 38 (1999), no. 1, 79-83]. Topology, 40(2):431-432, 2001. (cited on p. 2)

[Mat03] Daisuke Matsushita. Holomorphic symplectic manifolds and Lagrangian fibrations. Acta Appl. Math., 75(1-3):117-123, 2003. (cited on p. 2)

[WW03] Jan Wierzba and Jarosław A. Wiśniewski. Small contractions of symplectic 4-folds. Duke Math. J., 120(1):65-95, 2003. (cited on p. 10)

Daniel Greb, Institut fÜr Mathematik, Abteilung FÜr Reine MATHEMATiK, Albert-LudWigsUNIVERSITÄT FREIBURG, ECKERSTR. 1, 79104 FREIBURG IM BREISGAU, GERMANY

E-mail address: daniel.greb@math.uni-freiburg.de

CHRISTIAN LEHN, INSTITUt DE RECHERCHE MATHÉMATIQUe AVANCÉE, UniVERSitÉ DE STRASBOURG, 7 RUe René Descartes, 67084 Strasbourg Cedex, France

E-mail address: lehn@math.unistra.fr

SÖNKE ROLLENSKE, FAKULTÄT FÜR MATHEMATIK, UNIVERSTÄT BIELEFELD, UNIVERSITÄTSSTR. 25, 33615 BIELEFELD, GERMANY

E-mail address: rollenske@math.uni-bielefeld.de 